\title{
Faecal phytic acid and its relation to other putative markers of risk for colorectal cancer
}

\author{
R W Owen, U M Weisgerber, B Spiegelhalder, H Bartsch
}

\begin{abstract}
Aims-Phytic acid, a major constituent of cereals, pulses, and seeds has been advocated as an important antioxidant component of dietary fibre that affords possible protection against colorectal cancer. This is supported by experimental studies showing it has antineoplastic activity in animal models of both colon and breast cancer. To date the concentration of faecal phytic acid in human clinical groups has not been evaluated. Therefore the faecal phytic acid content of adenoma patients drawn from a placebo controlled calcium intervention trial was evaluated. Methods-Phytic acid was measured in faecal extracts by an improved ion-pair high performance liquid chromatography method.

Results-Phytic acid was detected in the range $0.68-4.00 \mu \mathrm{mol} / \mathrm{g}$ wet faeces and 55-2038 $\mu \mathrm{mol} / \mathrm{day}$. Linear regression analyses showed no association between stool phytic acid and lipid content. Strong correlations were seen, however, between phytic acid and iron content, both on a concentration $(r=0.52 ; p=0.00004)$ and daily excretion $\left(r=0.76 ; p=5.5 \times 10^{-12}\right)$ basis. Phytic acid was also strongly correlated with the daily excretion of calcium $\left(r=0.59 ; p=1.36 \times 10^{-6}\right)$ and magnesium $(r=0.42 ; p=0.001)$. Cell proliferation in the sigmoid colon, an intermediate biomarker of colorectal cancer was not significantly associated with faecal phytic acid, minerals or lipid content in this compromised clinical group.
\end{abstract}

Conclusions-This improved method, developed for the determination of phytic acid in faeces should allow further studies on the role of phytic acid in the aetiology of colorectal cancer to be conducted on a population or case control basis.

Divisions of Toxicology and Cancer Risk Factors $\mathrm{R}$ W Owen B Spiegelhalder H Bartsch

and Epidemiology U M Weisgerber

German Cancer Research Centre, Heidelberg, Germany

Correspondence to: Dr R W Owen, Division of Toxicology and Cancer Risk Factors, German Cancer Research Centre, Im Neuenheimer Feld 280, D-69120 Heidelberg, Germany.

Accepted for publication 31 October 1995 is a ubiquitous plant component, which constitute $1-5 \%$ by weight of most cereals, seeds is believed to be the reason for their remarkable longevity. The seeds of some plants may remain viable for up to 400 years. ${ }^{2}$ Graf and Eaton ${ }^{3}$ suggested that a major reason for this is that PA maintains iron in the Fe (III) oxidation state and obstructs generation of reactive oxygen species, for example, the hydroxyl radical (HO) thereby preventing oxidative damage, especially of unsaturated fatty acids, which are a major component of seeds.

The antioxidative properties of PA are ascribed to its chelating potential. Phytic acid exhibits a high affinity for all polyvalent cations and metal phytate complexes are well known to be insoluble over a wide $\mathrm{pH}$ range and this is particularly true for iron-phytate chelates. ${ }^{3}$ For this reason Graf and Eaton ${ }^{3}$ propose that all of the antioxidant properties of PA derive from its comparatively high binding affinity for iron. Iron catalysed reactions play a crucial part in oxidative damage to biological materials and can be generated by two commonly described mechanisms namely the Haber-Weiss and Fenton reactions.

For iron to participate in Fenton chemistry it must be chelated and soluble. The six coordination sites of trivalent iron, for example, $\mathrm{FeCl}_{3} \cdot 6 \mathrm{H}_{2} \mathrm{O}$ are occupied by water and hydroxide ions in aqueous solution. ${ }^{4}$ Most chelating agents displace five of these ligands and form a pentadentate chelate with $\mathrm{H}_{2} \mathrm{O}$ occupying the sixth coordination site. ${ }^{4}$ EDTA forms a hexadentate chelate but because of its small size distorts the chelate and makes a seventh site available for $\mathrm{H}_{2} \mathrm{O} .{ }^{4}$ Phytic acid apparently is unique in occupying six coordination sites and displacing all of the coordination water in the Fe-III-phytate complex. ${ }^{4}$ The lack of an aquo-coordination site has been shown spectrophotometrically with azide and confirmed by nuclear magnetic resonance determination. ${ }^{4}$ Therefore it has been proposed that PA preserves the solubility of iron making the metal totally unreactive. This is the presumed basis of the antioxidant capacity of PA and led Graf and Eaton ${ }^{5}$ to suggest that the PA content of the diet may be important in the modulation of colorectal cancer. Colorectal cancer is a disease of affluent societies and epidemiological studies $^{6-8}$ have clearly shown that dietary components are an important contributory factor. High consumption of fat and red meat are regarded important in the promotion of colorectal cancer perhaps through an additive effect on intestinal metabolism by increased hepatic secretion of biliary components and fermentation by the intestinal microflora. ${ }^{910}$ By contrast high consumption of dietary fibre is regarded ameliorative. ${ }^{11} 12$ The protective role of dietary fibre against colorectal cancer has its origins in the classic study of Burkitt. ${ }^{13}$ This was based on epidemiological studies in Africa, which showed that a high intake of dietary fibre was highly correlated with faecal 
weight. It was proposed that dietary fibre is protective against colorectal cancer by the simple mechanisms of stool bulking, acceleration of transit, and dilution of potential endogenous carcinogens. This theory has recently received qualified support in the metaanalyses of Howe et al ${ }^{14}$ and Cummings et al. ${ }^{15}$ Nevertheless certain inconsistencies in the dietary fibre data indicate that it may prove fruitful to analyse the PA content of dietary fibre and assess its effect on intestinal biochemistry.

The influence of PA in the genesis of colorectal cancer has received substantial support from animal model studies. Nielson et al ${ }^{16}$ studied the effect of adding PA at varying concentrations to the normal diet of the rat. Phytic acid at concentrations of $1 \cdot 2 \%$ and $2 \%$ significantly lowered colonic cell proliferation, an intermediate biomarker of colorectal cancer in these rats. Shamsuddin et al ${ }^{17-19}$ have shown in a series of studies utilising the azoxymethane rodent model that addition of PA $(1-2 \%)$ to the diet significantly reduces tumour burden and volume. The anticancer effect of PA was found to be dose dependent and temporal in that it significantly reduced the incidence and colon tumour volume when given five months after the last injection of azoxymethane.

Thompson and $\mathrm{Zhang}^{20}$ have shown that PA can reduce cell proliferation in both colonic and mammary tissues in the mouse and furthermore subverts the promotional effect of added iron and calcium. Nelson et al ${ }^{21}$ studied the combined effects of iron and PA and while the first of these was shown to promote 1,2dimethylhydrazine induced colorectal cancer in the rat these effects were nullified by simultaneous administration of the second.

Furthermore iron enriched diets caused an increase of tumour rate in two models of 1,2dimethylhydrazine induced colon tumorigenesis in mice. ${ }^{22} 23$ The effect was independent of the time the diet was fed, was dose dependent, and increased both tumour initiation and promotion.

Further support for the hypothesis of Graf and Eaton comes from studies on iron status and risk of cancer in humans. Stevens et $a^{24}$ reported that increased body iron stores were significantly associated with colon cancer in a national survey (NHANES 1) and this has been supported by a recent case control study ${ }^{25}$ showing that adenoma risk is positively associated with increased serum ferritin concentrations.

Despite all the circumstantial evidence, to date, no study has been conducted in the clinical domain to bring together the various factors of the phytic acid/reactive oxygen species hypothesis ${ }^{3}$ and its implications for cancer. Therefore cell proliferation rate, faecal lipid, mineral and PA content of patients with sporadic adenomatous polyps have been measured to establish any interrelation that may have a bearing on the aetiology of colorectal cancer.

A modified high performance liquid chromatography (HPLC) system was developed and used for PA analyses in selected foods and human faeces because of the unsatisfactory behaviour of those already published.

\section{Methods}

\section{Chemicals and reagents}

Phytic acid (40\% in water), formic acid, tetrabutylammonium hydroxide $(40 \%$ in water), tetrabutylammonium sulphate, and $\mathrm{FeCl}_{3} \cdot 6 \mathrm{H}_{2} \mathrm{O}$ were obtained from Aldrich Chemicals (Steinheim, Germany). Potassium and sodium phytate were obtained from Sigma Chemicals (Deisenhofen, Germany). The sodium salts of inositol hexaphosphate and pentaphospate were obtained from Calbiochem, La Jolla, California. All solutions were made up in millipore grade water.

\section{Study group}

The study group comprised 29 adenoma patients (Table I) drawn from a longterm placebo controlled calcium intervention study (unpublished data).

\section{Faecal samples}

Faecal samples were defecated into plastic bowls and placed immediately on dry ice before transportation to the laboratory. They were stored at $-80^{\circ} \mathrm{C}$ until further analyses. For aliquoting, samples were thawed overnight at $4^{\circ} \mathrm{C}$, pooled, and homogenised (room temperature) at $600 \mathrm{rpm}$ for five minutes in a rotating homogeniser (Heidolph, Kelheim, Germany). Aliquots of $40 \mathrm{~g}$ were freeze dried, reweighed, and ground to a fine homogeneous powder by mortar and pestle before the analyses of PA, lipids, and minerals. Remaining aliquots were refrozen at $-80^{\circ} \mathrm{C}$.

\section{Food samples}

The following foods were included in the study - namely, sesame seeds, wheat bran, peanuts, soy beans, coriander seeds, chilli seeds, pepper seeds, tomato seeds, and millett and were analysed after being lypholysed, pulverised by mortar and pestle to a fine homogeneous powder, and defatted by Soxhlet extraction for eight hours essentially as described by Fadden and Owen ${ }^{26}$ except that chloroform/methanol was replaced by pentane.

TABLE I Age, sex, and dietary data before intervention in the study groups

\begin{tabular}{lcc}
\hline & $\begin{array}{l}\text { Placebo group } \\
(n=15)\end{array}$ & $\begin{array}{l}\text { Calcium group } \\
(n=15)\end{array}$ \\
\hline Age & $63 \cdot 3(6(1)$ & $60 \cdot 6(9 \cdot 2)$ \\
No of female patients & 10 & 4 \\
Dietary intake & \\
Energy (kJ/d) & $10410(2169)$ & $11678(5788)$ \\
Protein (g/d) & $93(27)$ & $99(50)$ \\
Fat $(g / d)$ & $97(38)$ & $105(50)$ \\
Carbohydrates $(g / d)$ & $253(52)$ & $293(160)$ \\
Fibre $(g / d)$ & $31(7)$ & $35(20)$ \\
Calcium $(\mathrm{mg} / \mathrm{d})$ & $933(416)$ & $922(370)$ \\
Vitamin $D(\mu \mathrm{g} / \mathrm{d})$ & $4(4)$ & $4(3)$ \\
\hline
\end{tabular}

*As determined by food frequency questionnaire before intervention (adjusted for sex). Results expressed as mean (SD). 
Faeces $(500 \mathrm{mg})$

$0.5 \mathrm{M} \mathrm{HCl}$ at $25^{\circ} \mathrm{C}$

for two hours

Freeze overnight and centrifuge slurry at $5000 \times g$ for 20 minutes

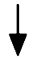

Evaporate to dryness

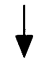

Dissolve in $0.01 \mathrm{M} \mathrm{HCl}(20 \mathrm{ml})$

$0.4 \mathrm{ml} / \mathrm{min}$

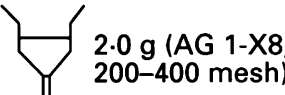

$\longrightarrow$ Discard eluate

With $20 \times 1 \mathrm{ml} 2 \mathrm{M} \mathrm{HCl}$

Elute inositol phosphates

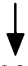

Evaporate to dryness, freeze-dry, and resuspend in distilled water

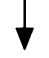

HPLC

Figure 1: Protocol for the analysis of phytic acid.

Sample extraction and preparation

Samples in the range $250-1000 \mathrm{mg}$ (routinely $250 \mathrm{mg}$ ) were extracted (Fig 1) with $20 \mathrm{ml} 0.5$ $\mathrm{M} \mathrm{HCl}$ under vigorous mechanical agitation $(500 \mathrm{rpm})$ for two hours at room temperature. ${ }^{27}$ The extracts were frozen overnight, thawed, centrifuged at $5000 \mathrm{rpm}$, and the supernatants were evaporated to dryness at $65^{\circ} \mathrm{C}$. The dried extracts were resuspended in $20 \mathrm{ml} 0.01 \mathrm{M} \mathrm{HCl}$ and percolated over Dowex anion-exchange gel $(1 \times 8,200-400$ mesh; $2 \mathrm{~g})$ in glass columns at a rate of $1 \mathrm{ml} /$ minute. The columns were washed with a further $40 \mathrm{ml} 0.01 \mathrm{M} \mathrm{HCl}$ before elution of PA with $20 \mathrm{ml} 2 \mathrm{M} \mathrm{HCl}$ at $0.4 \mathrm{ml} / \mathrm{min}$. The eluates were dried, resuspended in $20 \mathrm{ml}$ distilled water, filtered through MF-Millipore filters $(0 \cdot 2 \mu \mathrm{m})$, and redried. After freeze-drying overnight the extracts were resuspended in $1.0 \mathrm{ml}$ distilled water.

\section{Standards}

A stock PA solution was made by diluting $40 \%$ PA (Aldrich Chemical) in distilled water to give final concentrations in the range (1-60 $\mu \mathrm{mol} / \mathrm{ml})$. The linearity of PA concentration versus peak area was investigated by duplicate $20 \mu \mathrm{l}$ injections of each dilution. For comparison, standard curves were prepared with sodium phytate and potassium phytate (Sigma Chemical). The sodium salts of inositol pentaphosphate and hexaphosphate were also studied in this system:

A mixture of inositol phosphates was prepared by autoclaving PA $(20 \mathrm{ml})$ at $121^{\circ} \mathrm{C}$ for one hour and refluxing sodium phytate $(1 \mathrm{~g})$ in $100 \mathrm{ml} 0.5 \mathrm{M} \mathrm{HCl}$ for 16 hours. In the latter case, samples $(5.0 \mathrm{ml})$ were removed periodically to assess the degradation pattern. The samples were evaporated to dryness, freezedried, and resuspended in distilled water before injection into the HPLC.
HPLC analysis

Three different methods described in published works were compared for HPLC analyses of PA in biological material. The methods of Graf and Dintzis, ${ }^{27}$ Sandberg and Ahderrine, ${ }^{28}$ and Lee and Abendroth ${ }^{29}$ were applied exactly as described. For this study a modification of the method of Lee and Abendroth $^{29}$ was finally adopted as described below.

The mobile phase consisted of $0.005 \mathrm{M}$ tetrabutylammonium hydroxide in distilled water, $\mathrm{pH} 4.3$ (with formic acid, 6\%). Chromatography was conducted on a HewlettPackard HP 1090 liquid chromatograph fitted (routinely) with a C-18 (Latek, Eppelheim, Germany) reverse phase $25 \mathrm{~cm},(5 \mu)$ column (internal diameter; ID, $4.6 \mathrm{~mm}$ ) or else a C-18 (Supelco, Bad Homburg, Germany) reverse phase $25 \mathrm{~cm}(5 \mu)$ column (ID, $2 \cdot 1 \mathrm{~mm}$ ). Twenty $\mu$ l of sample was injected into the HPLC and PA was detected at $210 \mathrm{~nm}$ using a diode-array detector at room temperature. The optimal flow rate of the mobile phase was $1.0 \mathrm{ml} / \mathrm{min}$ and the column void volume was determined by injecting $\mathrm{NaCl}(20 \mu \mathrm{mol} / \mathrm{ml})$ dissolved in mobile phase. Instrument control and data handling was by means of a HP ChemStation operating in the Microsoft Windows software environment.

\section{Methods for determining labelling index, faecal lipids, and minerals}

Labelling index was measured as described previously by Weisgerber et al ${ }^{31}$ while faecal lipids and minerals were analysed by the methods of Owen et al. ${ }^{32-34}$

\section{Statistical analyses}

The interactions between PA, lipids, and minerals in faecal extracts were evaluated by multiple linear regression.

\section{Results}

Three different methods for the analysis of PA in biological material by HPLC were appraised. The method of Graf and Dintzis ${ }^{27}$ was found to be reproducible but as stated by the authors, PA eluted in the void volume and was prone to contamination by salts. Furthermore the PA peak was difficult to integrate because of considerable vacancy peaks at both the leading and trailing edges. The method of Sandberg and Ahderrine $^{28}$ on the other hand was found to be completely irreproducible. Using the exact conditions described PA, sodium phytate, potassium phytate, inositol pentaphosphate, and inositol hexaphosphate all eluted in the column void volume. This was the case when either a 4.6 or $2.1 \mathrm{~mm}$ (ID) C-18 column was used. Various permutations of the conditions described by Sandberg and Ahderrine ${ }^{28}$ such as varying the $\mathrm{pH}$, the proportion of methanol and tetrabutylammoniun hydroxide, substitution of tetrabutylammonium hydroxide with tetrabutylammonium sulphate had no effect on the retention time: PA in its various forms and 


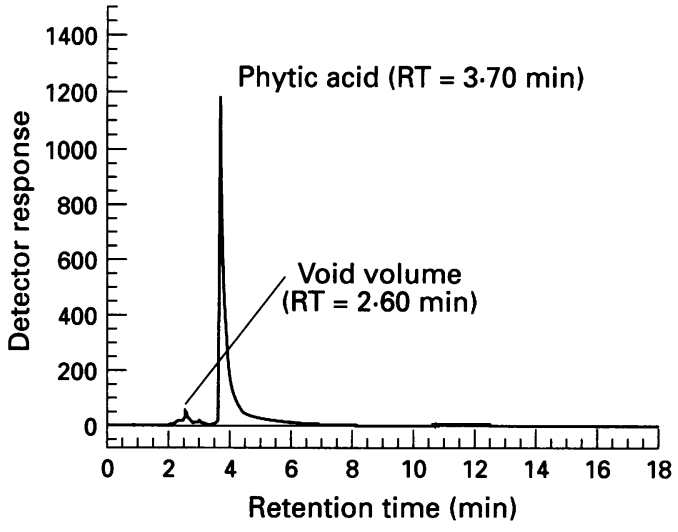

Figure 2: HPLC chromatogram of authentic phytic acid.

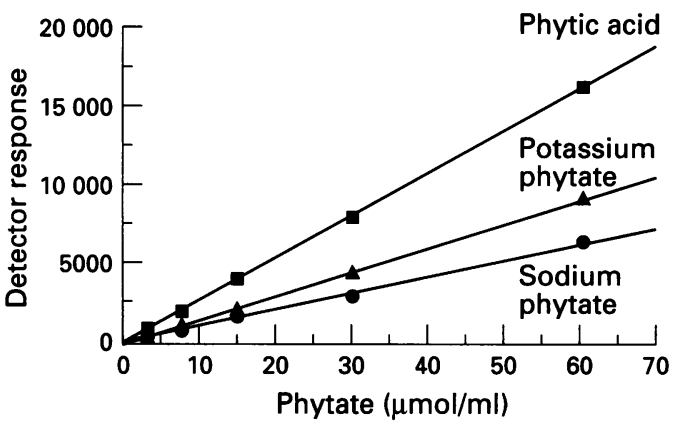

Figure 3: Phytate standard curves determined by HPLC.

inositol pentaphosphate always eluted in the void volume. The chromatography of $\mathrm{PA}$ in this system seems to be heavily dependent on the presence of methanol. When it was omitted from the mobile phase the PA became irretrievably bound to the column and could only be removed by the addition of methanol, which incidentally also simultaneously co-eluted tetrabutylammonium hydroxide making quantification of PA impossible.

When the method of Lee and Abendroth ${ }^{29}$ was used, PA was well separated from the void volume but peak shape was extremely poor. In this case variation of the conditions gave fruitful results. Stepwise lowering of the $\mathrm{pH}$ in increments of 0.5 units improved peak shape considerably with a simultaneous decrease in retention time. Optimum peak shape was obtained between $\mathrm{pH} 4-5$ and the final $\mathrm{pH}$ chosen was $4 \cdot 3$. Under the HPLC conditions described the void volume eluted at 2.6 minutes and PA at 3.7 minutes (Fig 2). The method was extremely reproducible and PA concentration versus peak area in the range

TABLE II Phytic acid (\%, dry weight) content of a range of foods

\begin{tabular}{lllll}
\hline & \multicolumn{2}{l}{ External standard } & \\
\cline { 2 - 4 } Food sample & Phytic acid & Sodium phytate & Sodium phytate & $\begin{array}{l}\text { Values from } \\
\text { published reports }\end{array}$ \\
\hline Sesame seeds & $2.21(0.02)$ & $7.83(0.07)$ & $5.56(0.05)$ & $5.36(0.09) \S$ \\
Wheat bran & $2 \cdot 28(0.08)$ & $8.14(0.37)$ & $5.78(0.22)$ & $5.03(1.85) \|$ \\
Peanuts & $0.86(0.02)$ & $3.05(0.07)$ & $2.17(0.04)$ & $1.88(0.05) \S$ \\
Soy beans & $0.89(0.06)$ & $3.09(0.22)$ & $2.20(0.16)$ & $1.84(0.03) \S$ \\
Tomato seeds & $1.24(0.14)$ & $4.36(0.49)$ & $3.10(0.35)$ & NP \\
Chilli seeds & $0.56(0.06)$ & $1.90(0.21)$ & $1.34(0.15)$ & NP \\
Coriander seeds & $1.11(0.03)$ & $3.93(0.10)$ & $2.79(0.07)$ & NP \\
Pepper seeds & $0.57(0.01)$ & $1.96(0.04)$ & $1.39(0.03)$ & NP \\
Millet & $0.18(0.01)$ & $0.62(0.03)$ & $0.44(0.02)$ & NP
\end{tabular}

^Analyses conducted as described in Methods. $\nmid$ Results expressed as \% (mean (SD)) of two to four samples: lyophilised, pulverised, and defatted with pentane (not corrected for defatting). † Sodium phytate corrected for salt content. Values from $§ G$ raf and Dintzis ${ }^{27}$ and $\|$ Camire and Clydesdale. ${ }^{30} \mathrm{NP}=$ not published.
TABLE III Faecal characteristics of the study group

\begin{tabular}{lcc}
\hline & \multicolumn{2}{l}{ Faecal excretion } \\
\cline { 2 - 3 } Faecal parameter & $\mu$ mol/g wet faeces & mmol/day \\
\hline Phytic acid & $1 \cdot 74(0.69)$ & $0.36(0 \cdot 19)$ \\
Iron & $2 \cdot 03(0.93)$ & $0.49(0 \cdot 49)$ \\
Calcium & $155(108)$ & $34(27)$ \\
$\quad$ Total & $19(12)$ & $4 \cdot 46(3 \cdot 58)$ \\
Soluble & $31(31)$ & $6 \cdot 85(7 \cdot 02)$ \\
Soaps & $74(35)$ & $15(11)$ \\
Magnesium & $125(45)$ & $26(13)$ \\
Phosphate & $4 \cdot 52(2.60)$ & $0.94(0 \cdot 60)$ \\
Bile acids & $50(30)$ & $11(11)$ \\
Long chain fatty acids & $11(6)$ & $2 \cdot 71(3.37)$ \\
Neutral sterols & $3.23(2.92)$ & $0.68(0 \cdot 72)$ \\
Plant sterols & $6.55(0 \cdot 42)$ & $\mathrm{NA}$ \\
pH & $11.86(3.99)$ & $\mathrm{NA}$ \\
Labelling index (\%) & $\mathrm{NA}$ & $225(107)$ \\
Faecal wet weight (g) & & \\
\hline
\end{tabular}

Data expressed as mean $(\mathrm{SD})(\mathrm{n}=29)$. NA=not applicable.

1-60 $\mu \mathrm{mol} / \mathrm{ml}$ was found to be linearly proportional over the entire range (Fig 3 ). The peak areas of sodium and potassium phytate (Fig 3) and sodium inositol pentaphosphate and inositol hexaphosphate gave similar linear functions but of a lesser magnitude because of a lower proportional content of actual PA. The PA and sodium phytate digests showed that the lower inositol phosphates, like authentic inositol pentaphosphate could not be separated from PA or inositol hexaphosphate in this system. This method therefore groups all the inositol phosphates as PA and gives a value for total phytate in biological samples compared with the published method of Sandberg and Ahderrine, ${ }^{28}$ which described earlier could not be reproduced.

Analysis of PA in selected foodstuffs showed a good correlation with other methods but in general the values obtained were lower (Table II) when calculated against free PA as external standard. When calculation was made against a sodium phytate standard curve (with correction for sodium content), however, the data were very close to previously published values. The data show a similar trend to previous studies in that the highest PA content of those compared was found in wheat bran and the lowest in soy beans. Phytic acid concentrations were also determined in foodstuffs, which have not been previously reported and of these coriander, tomato, and green pepper seeds were found to contain appreciable amounts (Table II). Efficiency of extraction was dependent on the foodstuff to solvent ratio and decreased significantly $(r=-0.97 ; \mathrm{p}=0.03)$

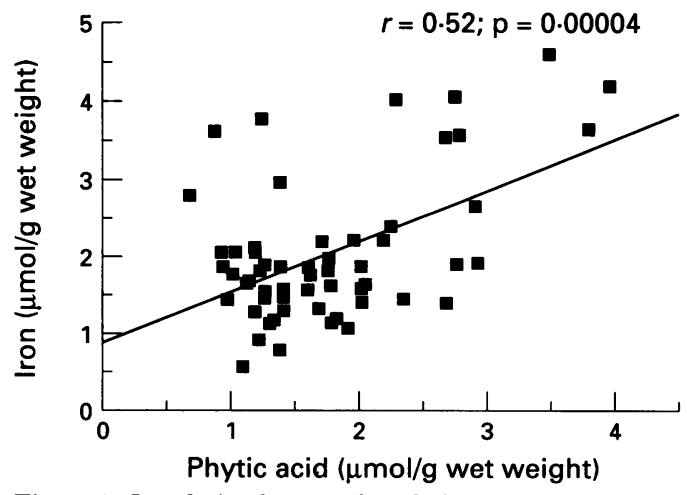

Figure 4: Correlation between faecal phytic acid and iron excretion. 


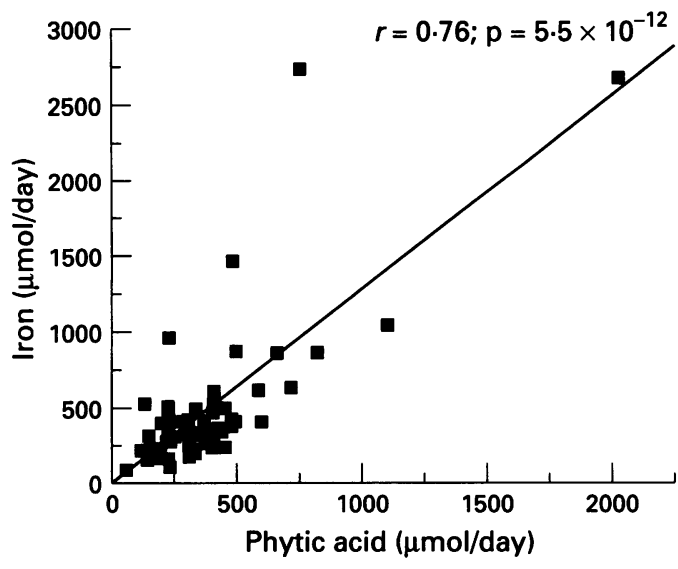

Figure 5: Correlation between daily faecal phytic acid and iron excretion.

between 1:80 and 1:20 as determined with a concentration range of defatted sesame seeds (data not shown).

The HPLC method was also found to be appropriate for the measurement of PA in human faeces. The acid extracts of the adenoma patients contained appreciable amounts of proteinacous material ( $\lambda$ maximum 240 $280 \mathrm{~nm}$ as determined by HPLC) but this was removed completely by the prior anionexchange chromatography step.

Phytic acid was detected in faecal extracts of the adenoma patients in the range 0.68 $4.00 \mu \mathrm{mol} / \mathrm{g}$ wet faeces and 55-2038 $\mu \mathrm{mol} /$ day (Table III). There was little difference in the mean (SD) excretion of PA in the placebo $(1.88(0.80)$ v $1.78(0.67) \mu \mathrm{mol} / \mathrm{g})$ and calcium $(1.60 \quad(0.59) \quad v \quad 1.86 \quad(0.88)$ $\mu \mathrm{mol} / \mathrm{g}$ ) arms of the composite groups both before and after intervention. Linear regression analyses of PA versus faecal lipid and mineral content and intestinal cell proliferation (Table III) showed the following. The amount of PA in the stool was strongly correlated (Fig 4) with faecal iron $(r=0.52 ; \mathrm{p}=0.00004)$, unsaturated fatty acids $(r=0.35 ; \mathrm{p}=0.004)$, and total calcium content of the stool $(r=0.34 ; \mathrm{p}=0.01)$. A weak positive association was seen between PA and magnesium $(r=0.26 ; p=0.04)$. The association between PA and minerals was even stronger when analysed on a daily basis: PA $v$ iron, $r=0.76 ; \mathrm{p}=5.5 \times 10^{-12}$ (Fig 5): PA $v$ total calcium, $r=0.59 ; \mathrm{p}=1.36 \times 10^{-6}$ (Fig 6): PA $v$ magnesium, $r=0.42 ; \mathrm{p}=0.001$. No association existed between stool PA content and the

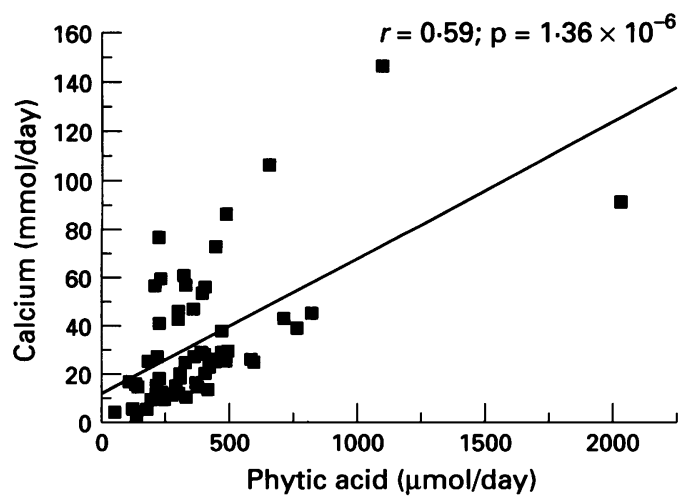

Figure 6: Correlation between daily faecal phytate and calcium excretion.

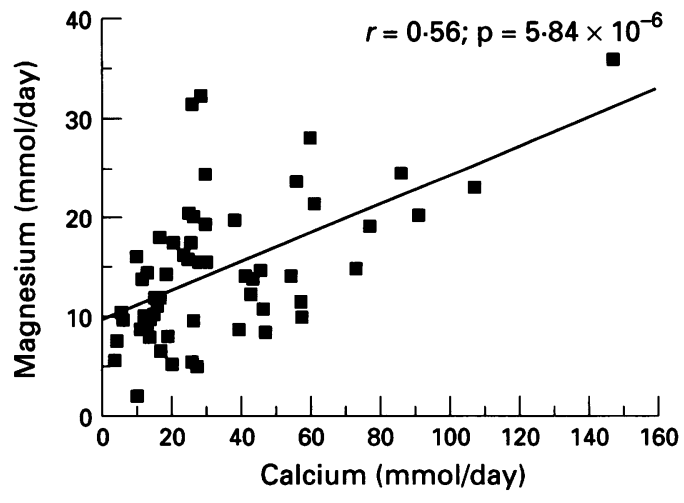

Figure 7: Correlation between daily faecal magnesium and calcium excretion.

concentration and daily excretion of phosphate, bile acids, long chain fatty acids, neutral sterols, and plant sterols.

Intermineral positive associations were also seen. The faecal excretion of calcium and magnesium was positively correlated on both a concentration $(r=0.36 ; \mathrm{p}=0.006)$ and daily $\left(r=0.56 ; p=5.84 \times 10^{-6}\right)$ basis (Fig 7).

Cell proliferation, an intermediate biomarker of colorectal cancer showed no association with faecal PA $(r=0 \cdot 17 ; \mathrm{p}=0 \cdot 20)$ or with faecal iron $(r=-0 \cdot 18 ; \mathrm{p}=0 \cdot 17)$. It was, however, positively correlated with faecal magnesium $(r=0.28 ; \mathrm{p}=0.04)$ concentration. Cell proliferation was not correlated with faecal total calcium concentration taking the study group as a whole $(r=0.05 ; p=0.69)$ or when comparison was adjusted for calcium intervention, but, was positively correlated with soluble calcium $(r=0.30 ; p=0.03)$. Faecal soluble calcium (Fig 8) was found to be strongly associated with total calcium concentration $(r=0.51$; $\mathrm{p}=0 \cdot 00005$ ). Cell proliferation did not correlate with the faecal concentration of phosphate, bile acids, long chain fatty acids, neutrals sterols or calcium-long chain fatty acid soaps and on a daily basis no association apart for magnesium $(r=0.30 ; p=0.03)$ was found with any of the faecal characteristics. Furthermore there was no association between the faecal concentration of bile acids in relation to long chain fatty acids and neutral sterols.

\section{Discussion}

A reproducible HPLC method has been developed and validated that enables the accurate quantification of PA in faeces. The method,

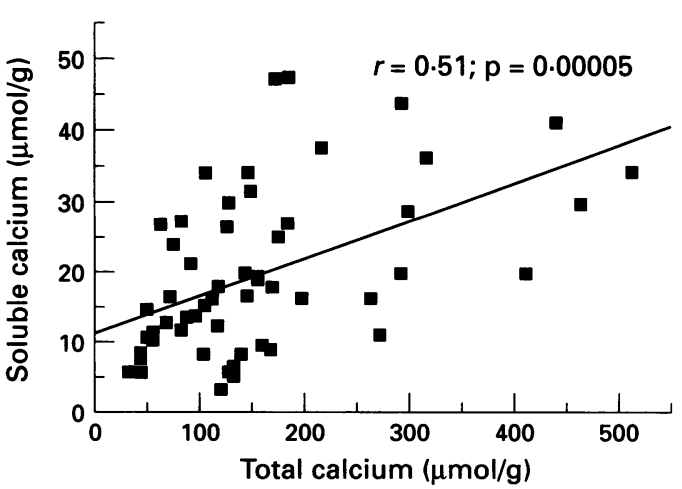

Figure 8: Correlation between faecal soluble calcium and total calcium. 
based on that described by Lee and Abendroth, ${ }^{29}$ is not over complex and has been successfully applied to the analyses of PA in foodstuffs and faecal specimens in patients with sporadic adenoma. The method gives a value for total PA in biological material and as yet cannot be used to resolve the lower inositol phosphates, for example, inositol triphosphateinositol pentaphosphate from PA as described by Sandberg and Ahderrine. ${ }^{28}$ Despite rigorous adherence to the method conditions described by this last group, it could not be reproduced, and all attempts to modify it were in vain. When methanol was omitted from the mobile phase, PA was not eluted after several hours. Addition of methanol up to $55 \%$ either as increments $(5 \%)$, or as a linear gradient removed PA from the column but it always coeluted with salts and tetrabutylammonium hydroxide. The crux of the problem here is that in the presence of methanol, tetrabutylammonium hydroxide is unable to bind to C-18 reverse-phase columns and ion-pair chromatography in this system is impossible to achieve. The reasons for this discrepancy with the published method of Sandberg and Ahderrine ${ }^{28}$ remain in part unexplained.

Authentic free PA was well resolved from the void volume with excellent peak shape using the current method. Free PA in comparison to potassium phytate gave rise to a small peak in the void volume while potassium phytate gave a significant void volume peak due to dissociation of $\mathrm{K}^{+}$and PA in the mobile phase. Sodium phytate and sodium inositol pentaphosphate and hexaphosphate eluted in the void volume and dissociation and resolution of the PA from the void volume could only be achieved by a prior anion-exchange chromatography step. Probably the sodium salts $\left(\mathrm{Na}_{12}\right)$ of PA and the lower inositol phosphates when solubilised in mobile phase at pH 4.3 are not dissociated and therefore PA cannot participate in ion-pair chromatography. How successful HPLC of authentic sodium salts of PA and its derivatives has been achieved as described by various groups remains unexplained because if PA does not dissociate from the salt then ion-pair chromatography with tetrabutylammonium hydroxide is not possible.

The PA content of various foodstuffs obtained in this system compares favourably with other published methods. The values obtained were in general a little lower but as the comparable published values were obtained by the HPLC system of Graf and Dintzis $^{27}$ and iron precipitation methods perhaps this is not surprising.

When the method was applied to the analysis of faeces from adenoma patients PA was detected in the range $0.68-4.0 \mu \mathrm{mol} / \mathrm{g}$ wet material (55-2038 $\mu \mathrm{mol} /$ day) with little difference in mean values both before and after intervention. This range when compared on a dry weight basis is roughly comparable to the limited examples published for human and rat faeces. ${ }^{35}$

This shows that the subjects included in this high risk study group consumed considerable amounts of PA, because it is generally regarded that the faecal profile is an adequate mirror of this for the following reasons. Studies in humans and pigs show that approximately $60-70 \%$ of ingested PA passes undergraded to faeces after gastrointestinal transit despite indications that some foodstuffs ${ }^{36}$ and the small intestine ${ }^{37}$ of humans and rats contain appreciable phytase activity.

The data showed a striking correlation especially on a daily basis between the faecal content of PA and iron, vindicating the hypothesis that PA may be important in binding of this ion and preventing its absorption and possible participation in reactive oxygen species generating mechanisms in the body. The notion that high consumption of PA may contribute to excessive mineral $\operatorname{loss}^{38}$ was supported by the fact that it was positively associated with faecal excretion of calcium and magnesium. This was particularly true for calcium when adjustment was made for calcium intervention.

Much of the circumstantial evidence and especially that obtained from animal model studies shows that PA through its ability to chelate iron could play an important part in the chemoprevention of colorectal cancer. Therefore it was important to assess faecal PA content in relation to cell proliferation of the intestinal mucosa. However, no association between cell proliferation and stool PA and iron on both a concentration and daily excretion basis was seen. It should be noted, however, that in this clinical study the early events of colonic neoplasia had already occurred (that is, adenoma formation) and it would have been unlikely that stool parameters such as PA and iron concentration would have displayed correlations with cell proliferation because the presence of adenomas is known to have considerable influence on cell turnover in the colorectum. ${ }^{39}$

In conclusion a method has been developed for the reliable determination of PA in human faeces. Stool PA content shows a strong correlation with the excretion of minerals such as iron, calcium, and magnesium but exhibits no relation with cell proliferation in this clinical group. Similar studies on a population and case control basis are warranted, however, to fully assess the role of PA in colorectal carcinogenesis.

1 Maga JA. Phytate; its chemistry, occurrence, food interactions, nutritional significance and methods of analysis f Agric Food Chem 1982; 30: 1-9.

2 Ohlrogge JB, Kernan TP. Oxygen dependent ageing of seeds. Plant Physiol 1982; 70: 791-4.

3 Graf E, Eaton JW. Antioxidant functions of phytic acid. Free Radic Biol Med 1990; 8: 61-9.

4 Graf E, Mahoney JR, Bryant RG, Eaton JW. Iron catalysed hydroxyl radical formation: stringent requirement for free hydroxyl radical formation: stringent requirement for free
iron coordination site. $\mathcal{F}$ Biol Chem 1984; 259: 3620-4. 5 Graf E, Eaton JW. Dietary suppression of colonic cancer. Fraf E, Eaton JW. Dietary suppression of

6 Armstrong B, Doll R. Environmental factors and cancer Armstrong B, Doll R. Environmental factors and cancer
incidence and mortality in different countries, with special incidence and mortality in different countries, with special
reference to dietary practices. Int $f$ Cancer 1975; 15: reference

7 Willett WC, Stampfer MJ, Colditz GA, Rosner BA, Speizer FE. Relation of meat, fat and fiber intake to the risk of colon cancer in a prospective study among women. $N$ Eng f Med 1990; 323: 1664-72.

8 Giovanucci E, Rimm EB, Stampfer MJ, Colditz GA Ascherio A, Willett WC. Intake of fat, meat and fiber in relation to risk of colon cancer in men. Cancer Res 1994; 54: $2390-7$. 
9 Hill MJ, Drasar BS, Williams REO, Meade TW, Cox AG, Simpson JEP, et al. Faecal bile acids and clostridia in patients with cancer of the large bowel. Lancet 1975; i 535-9.

10 Reddy BS, Wynder EL. Metabolic epidemiology of colon cancer: fecal bile acids and neutral sterols in colon cance patients and patients with adenomatous polyps. Cancer 1977; 39: 2533-9.

11 Phillips SF, Pemberton JH, Shorter RG, eds. The large intestine: physiology, pathophysiology and disease. New York: Raven, 1991: 52-92

12 Spiller GA, ed. CRC handbook of dietary fiber in human nutrition. Boca Raton, FL: CRC Press, 1986: 211-80.

13 Burkitt DP. Epidemiology of cancer of the colon and rectum. Cancer 1971; 28: 3-13.

14 Howe GR, Benito E, Castelleto R, Cornee J, Esteve J Gallagher RP, et al. Dietary intake of fiber and decreased risk of cancers of the colon and rectum: evidence from the combined analysis of 13 case-control studies. If Nat Cancer Inst 1992; 84: 1887-96.

15 Cummings JH, Bingham SA, Heaton KW, Eastwood MA Faecal weight, colon cancer risk and dietary intake of nonstarch polysaccharides (dietary fiber), Gastroenterology 1992; 103: 1783-9.

16 Nielson BK, Thompson LU, Bird RP. Effect of phytic acid on colonic cell proliferation. Cancer Lett 1987; 37: $317-25$.

17 Shamsuddin AM, Elsayed AM, Ullah A. Suppression of large intestinal cancer in F344 rats by inositol hexaphosphate. Carcinogenesis 1988; 9: 577-80.

18 Shamsuddin AM, Ullah A. Inositol hexaphosphate inhibits arge intestinal cancer in F344 rats 5 months after induction by azoxymethane. Carcinogenesis 1989; 10: 625-6.

19 Ullah A, Shamsuddin AM. Dose-dependent inhibition of large intestinal cancer by inositol hexaphosphate in F344 rats. Carcinogenesis 1990; 11: 2219-22.

20 Thompson LU, Zhang L. Phytic acid and minerals: effect on early markers of risk for mammary and colon carcinogenesis. Carcinogenesis 1991; 11: 2041-5.

21 Nelson RL, Yoo SJ, Tanure JC, Andrianopoulos S, Misumi A. The effect of iron on experimental colorectal carcinog. Thesis. Anticancer Res 1989; 9: 1477-82.

22 Siegers C-P, Bumann D, Baretton G, Younes M. Dietary iron enhances the tumor rate in dimethylhydrazineinduced colon carcinogenesis in mice. Cancer Lett 1988; 41: 251-6.

23 Siegers C-P, Bumann D, Trepkan HD, Schadwinkel B, Baretton G. Influence of dietary iron overload on cell proliferation and intestinal tumorigenesis in mice. Cancer Lett 1992; 65: 245-9.

24 Stevens RG, Jones DY, Micozzi MS, Taylor PR. Body iron stores and risk for cancer. $N$ Engl $\mathcal{F}$ Med 1988; 319; $1047-52$.
25 Nelson RL, Davis FG, Satter E, Sobin LH, Kikendall W, Bowen P. Body iron stores and risk of colonic neoplasia. 7 Natl Cancer Inst 1994; 86: 455-60.

26 Fadden K, Owen RW. Faecal steroids and colorectal cancer: the effect of lactulose on faecal bacterial metabolism in a continuous culture model of the large intestine. Eur 7 Cancer Prev 1992; 1: 113-27.

27 Graf E, Dintzis FR. High performance liquid chromatographic method for the determination of phytate. Anal Biochem 1982; 119: 413-7.

28 Sandberg A-S, Ahderrine R. HPLC method for determination of inositol tri-, tetra-, penta- and hexaphosphates in foods and intestinal contents. F Food Sci 1986; 51: foods and

29 Lee K, Abendroth JA. High performance liquid chromatographic determination of phytic acid in foods. $\mathcal{f}$ Food $\mathrm{Sc}$ 983; 48: 1344-51.

30 Camire AL, Clydesdale FM. Analysis of phytic acid in foods by HPLC. I Food Sci 1982; 47: 575-8.

31 Weisgerber UM, Boeing H, Nemitz R, Raedsch R, Waldherr R. Proliferation cell nuclear antigen (clone 19A2) correlates with 5-bromo-2-deoxyuridine labelling in human colonic epithelium. Gut 1993; 34: 1587-92.

32 Owen RW, Thompson MH, Hill MJ. Analysis of metabolic prolifes of steroids in faeces of healthy subjects undergoing chenodeoxycholic acid treatment by liquid-gel chromatography and gas-liquid chromatography-mass chromatography and gas-liquid chromatography-

33 Costa NM, Low AG, Walker AF, Owen RW, Englyst HN. Effect of baked beans (Phaseolus vulgaris) on steroid metabolism and non-starch polysaccharide output of hypercholesterolaemic pigs with or without an ileo-rectal anastomosis. Br $\mathcal{F}$ Nutr 1994; 71: 871-86.

34 Owen RW, Weisgerber UM, Carr J, Harrison MH. Analysis of calcium lipid complexes in faeces. Eur f Cancer Prev 1995; 4: 247-55.

35 Knuckles BE, Kuzimicky DD, Betschart AA. HPLC analysis of phytic acid in selected foods and biological samples. IFood Sci 1982; 47: 1257-62.

36 Sandberg A-S, Svanberg U. Phytate hydrolysis by phytase in cereals, effects on in vivo estimation of iron availability. cereals, effects on in vivo estim

37 Iqbal TH, Lewis KO, Cooper BT. Phytase activity in the human and rat intestine. Gut 1994; 35: 1233-6.

38 Torre M, Rodriguez AR, Saura-Calixto F. Effects of dietary fiber and phytic acid on mineral availability. Crit Rev Food Sci Nutr 1991; 1: 1-22.

39 Risio M, Lipkin M, Candelaresi G, Bertone A, Coverlizza S, Rossini FP. Correlations between rectal mucosa cell proliferation and the clinical and pathological features of nonfamilial neoplasia of the large intestine. Cancer Res 1991; 51: $1917-21$. 